\title{
Adaptive Semi-Blind Space-Time Equalisation for Frequency Selective Rayleigh Fading MIMO Systems
}

\author{
Huiting Cheng ${ }^{\dagger}$, Sheng Chen ${ }^{\ddagger}$ and Yasushi Yamao $^{\dagger}$ \\ ${ }^{\dagger}$ Advanced Wireless Communication Research Center, University of Electro-Communications, Tokyo, Japan \\ ${ }^{\ddagger}$ School of Electronics and Computer Science, University of Southampton, Southampton SO17 1BJ, UK
}

\begin{abstract}
An adaptive semi-blind space-time equaliser (STE) has recently been proposed based on a concurrent gradientNewton constant modulus algorithm and soft decision-directed scheme for dispersive multiple-input multiple-output (MIMO) systems that employ high-throughput quadrature amplitude modulation signalling. We investigate the performance of this adaptive semi-blind STE operating in Rayleigh fading MIMO systems. Our results obtained show that the tracking performance of this semi-blind adaptive algorithm is close to that of the training-based recursive least squares algorithm. This study, therefore, demonstrates that the proposed semi-blind algorithm offers a practical means to adapt a STE in the hostile dispersive Rayleigh fading MIMO environment.
\end{abstract}

\section{INTRODUCTION}

Multiple-input multiple-output (MIMO) techniques are capable of offering a high channel capacity in interferencefree scenarios, but their achievable performance is limited by the multi-user interference. For frequency selective MIMO systems, space-time equalisers (STEs) [1]-[7] offer an effective means of suppressing both intersymbol interference and co-channel interference. To further improve the achievable bandwidth efficiency, high-throughput quadrature amplitude modulation (QAM) schemes [8] have become popular in numerous wireless network standards. For example, the 16QAM and 64-QAM schemes were adopted in the recent WiMax standard [9]. Adaptive implementation of STE can be realised using training based adaptive algorithms, such as the recursive least squares (RLS) algorithm [10]. However, a large number of training symbols is required to properly train a STE, which considerably reduces the achievable system throughput. Under dispersive MIMO environments, the STE's input signal is highly correlated and the stochasticgradient (SG) based adaptive algorithms, such as the trainingbased least mean square algorithm, suffers from slow convergence and high steady-state misadjustment [10].

Blind adaptive methods do not require training symbols and, therefore, do not reduce the achievable system throughput. However, pure blind adaptive STEs impose high complexity and suffer from slow convergence. Moreover, they result in unavoidable estimation and decision ambiguities [11], [12]. An effective means of resolving these ambiguities is to employ a few training symbols, leading to the attractive semiblind schemes. Many SG-based adaptive semi-blind methods [13]-[19] have been proposed for frequency nonselective MIMO systems. In particular, the work of [19] has developed a SG-based concurrent constant modulus algorithm (CMA) and soft decision-directed (SDD) scheme for narrowband MIMO systems that employ high-order QAM signalling. A SG-based semi-blind adaptive scheme however suffers from slow convergence and high steady-state misadjustment, when operating in frequency selective MIMO systems.

Recently, a gradient-Newton (GN) semi-blind concurrent CMA and SDD algorithm [20] was proposed to adapt the STE that operates in frequency selective MIMO systems. For stationary MIMO systems, the results reported in [20] have demonstrated that this semi-blind GN-CMA+SDD adaptive STE converges to the optimal minimum mean square error (MMSE) STE solution at a fast rate that is very close to the training-based RLS algorithm. No result however has been produced for this semi-blind GN-CMA+SDD based adaptive STE operating in time-varying MIMO channels. The contribution of this paper is that we investigate the tracking performance of this adaptive semi-blind STE operating in dispersive Rayleigh fading MIMO systems. Our results show that the tracking performance of this semi-blind adaptive algorithm is close to that of the continuously training-based RLS algorithm. Considering the fact that the continuously training-based RLS STE is impossible to realise and its symbol error rate (SER) offers a low bound of the system's achievable performance, this demonstrates that the semiblind GN-CMA+SDD algorithm offers a practical means to adapt a STE in the hostile dispersive fading MIMO environment.

\section{System Model And STE Structure}

Consider the space-division multiple-access (SDMA) induced MIMO system, where each of the $Q$ users is equipped with a single transmit antenna and the receiver is assisted by a $P$-element antenna array. Denote the symbol-rate channel impulse response (CIR) connecting the $q$ th transmit antenna to the $p$ th receive antenna at the symbol index $k$ as

$$
\mathbf{c}_{p, q}(k)=\left[c_{0, p, q}(k) c_{1, p, q}(k) \cdots c_{n_{C}-1, p, q}(k)\right]^{\mathrm{T}},
$$

where for notational simplicity we have assumed that each of the $P \times Q$ CIRs has the same length of $n_{C}$. Magnitudes of the CIR taps are uncorrelated Rayleigh processes, and each CIR tap has a root mean power of $\sqrt{0.5}+j \sqrt{0.5}$. The normalised Doppler frequency of the system is denoted by $f_{d}$, and continuously fluctuating fading is assumed, which provides a different fading magnitude and phase for each 
CIR tap $c_{i, p . q}$ at each $k$. The symbol-rate received signal samples $x_{p}(k), 1 \leq p \leq P$, can be expressed as

$$
x_{p}(k)=\sum_{q=1}^{Q} \sum_{i=0}^{n_{C}-1} c_{i, p, q}(k) s_{q}(k-i)+n_{p}(k),
$$

where $n_{p}(k)$ is a complex-valued Gaussian white noise process with $E\left[\left|n_{p}(k)\right|^{2}\right]=2 \sigma_{n}^{2}, s_{q}(k)$ is the $k$ th transmitted symbol of user $q$ with the symbol energy $E\left[\left|s_{q}(k)\right|^{2}\right]=\sigma_{s}^{2}$, and $s_{q}(k)$ takes the values from the $M$-QAM symbol set

$$
\mathcal{S} \triangleq\left\{s_{i, l}=u_{i}+j u_{l}, 1 \leq i, l \leq \sqrt{M}\right\}
$$

with the real-part symbol $\Re\left[s_{i, l}\right]=u_{i}=2 i-\sqrt{M}-1$ and the imaginary-part symbol $\Im\left[s_{i, l}\right]=u_{l}=2 l-\sqrt{M}-1$. The average signal-to-noise ratio (SNR) is defined as

$$
\mathrm{SNR}=\frac{\sum_{q=1}^{Q} \sum_{p=1}^{P} E\left[\mathbf{c}_{p, q}^{\mathrm{H}}(k) \mathbf{c}_{p, q}(k)\right] \sigma_{s}^{2}}{2 Q P \sigma_{n}^{2}}=\frac{n_{C} \sigma_{s}^{2}}{2 \sigma_{n}^{2}} .
$$

The STE for detecting the $q$ th user's data is given by

$$
y_{q}(k)=\sum_{p=1}^{P} \sum_{i=0}^{D-1} w_{i, p, q}^{*}(k) x_{p}(k-i),
$$

which is used to produce an estimate $\hat{s}_{q}\left(k-\tau_{q}\right)$ of the transmitted $s_{q}\left(k-\tau_{q}\right)$, where $D$ is the temporal filter's length, $w_{i, p, q}(k)$ are the weights of the STE at the symbol index $k$, and $0 \leq \tau_{q} \leq D+n_{C}-2$ is the decision delay.

Define the overall received signal vector $\mathbf{x}(k)=$ $\left[\mathbf{x}_{1}^{\mathrm{T}}(k) \mathbf{x}_{2}^{\mathrm{T}}(k) \cdots \mathbf{x}_{P}^{\mathrm{T}}(k)\right]^{\mathrm{T}}$, where

$$
\mathbf{x}_{p}(k)=\left[x_{p}(k) x_{p}(k-1) \cdots x_{p}(k-D+1)\right]^{\mathrm{T}},
$$

for $1 \leq p \leq P$. Then $\mathbf{x}(k)$ can be expressed by the wellknown MIMO model

$$
\mathbf{x}(k)=\mathbf{C}(k) \mathbf{s}(k)+\mathbf{n}(k)
$$

where $\mathbf{n}(k)=\left[\mathbf{n}_{1}^{\mathrm{T}}(k) \mathbf{n}_{2}^{\mathrm{T}}(k) \cdots \mathbf{n}_{P}^{\mathrm{T}}(k)\right]^{\mathrm{T}}$ with

$$
\mathbf{n}_{p}(k)=\left[n_{p}(k) n_{p}(k-1) \cdots n_{p}(k-D+1)\right]^{\mathrm{T}}
$$

for $1 \leq p \leq P$, the transmitted symbol vector of all the users $\mathbf{s}(k)=\left[\mathbf{s}_{1}^{\mathrm{T}}(k) \mathbf{s}_{2}^{\mathrm{T}}(k) \cdots \mathbf{s}_{Q}^{\mathrm{T}}(k)\right]^{\mathrm{T}}$ with

$$
\mathbf{s}_{q}(k)=\left[s_{q}(k) s_{q}(k-1) \cdots s_{q}\left(k-D-n_{C}+2\right)\right]^{\mathrm{T}},
$$

for $1 \leq q \leq Q$, and the overall system's CIR matrix

$$
\mathbf{C}(k)=\left[\begin{array}{cccc}
\mathbf{C}_{1,1}(k) & \mathbf{C}_{1,2}(k) & \cdots & \mathbf{C}_{1, Q}(k) \\
\mathbf{C}_{2,1}(k) & \mathbf{C}_{2,2}(k) & \cdots & \mathbf{C}_{2, Q}(k) \\
\vdots & \vdots & \cdots & \vdots \\
\mathbf{C}_{P, 1}(k) & \mathbf{C}_{P, 2}(k) & \cdots & \mathbf{C}_{P, Q}(k)
\end{array}\right]
$$

with the $D \times\left(D+n_{C}-1\right)$ CIR matrix associated with the user $q$ and the receive antenna $p$ given by the Toeplitz form

$$
\mathbf{C}_{p, q}(k)=\left[\begin{array}{cccc}
\mathbf{c}_{p, q}^{\mathrm{T}}(k) & 0 & \cdots & 0 \\
0 & \mathbf{c}_{p, q}^{\mathrm{T}}(k) & \ddots & \vdots \\
\vdots & \ddots & \ddots & 0 \\
0 & \cdots & 0 & \mathbf{c}_{p, q}^{\mathrm{T}}(k)
\end{array}\right]
$$

for $1 \leq p \leq P$ and $1 \leq q \leq Q$. Similarly, the STE for detecting the $q$ th user's data can be expressed as

$$
y_{q}(k)=\mathbf{w}_{q}^{\mathrm{H}}(k) \mathbf{x}(k)
$$

where the overall weight vector of the STE at $k$ is given by $\mathbf{w}_{q}(k)=\left[\mathbf{w}_{1, q}^{\mathrm{T}}(k) \mathbf{w}_{2, q}^{\mathrm{T}}(k) \cdots \mathbf{w}_{P, q}^{\mathrm{T}}(k)\right]^{\mathrm{T}}$ with

$$
\mathbf{w}_{p, q}(k)=\left[w_{0, p, q}(k) w_{1, p, q}(k) \cdots w_{D-1, p, q}(k)\right]^{\mathrm{T}} .
$$

The dimension of the STE is therefore $N_{\mathrm{STE}}=P \cdot D$.

\section{SEMI-BLIND GN-CMA+SDD ALGORITHM}

Let the number of available training symbols be $K$, and denote the available training data as

$$
\left\{\begin{array}{l}
\mathbf{X}_{K}=[\mathbf{x}(1) \mathbf{x}(2) \cdots \mathbf{x}(K)], \\
\overline{\mathbf{s}}_{K, q}=\left[s_{q}\left(1-\tau_{q}\right) s_{q}\left(2-\tau_{q}\right) \cdots s_{q}\left(K-\tau_{q}\right)\right]^{\mathrm{T}} .
\end{array}\right.
$$

The least squares (LS) estimate of the STE's weight vector based on $\left\{\mathbf{X}_{K}, \overline{\mathbf{s}}_{K, q}\right\}$ is readily given as

$$
\mathbf{w}_{q}(0)=\left(\mathbf{X}_{K} \mathbf{X}_{K}^{\mathrm{H}}\right)^{-1} \mathbf{X}_{K} \overline{\mathbf{s}}_{K, q}^{*} .
$$

In order to maintain throughput, the number of training pilots should be as small as possible. To ensure that $\mathbf{X}_{K} \mathbf{X}_{K}^{\mathrm{H}}$ has a full rank, on the other hand, $K$ should be chosen to be slightly larger than $N_{\mathrm{STE}}$, the dimension of $\mathbf{x}(k)$. Because the training data with $K \approx N_{\mathrm{STE}}$ are generally insufficient, the initial LS weight vector (15) may not be sufficiently accurate to open the eye. Therefore, decision direct adaptation is generally unsafe. Also directly applying the SGCMA+SDD blind scheme of [21] to adapt the STE (12) with $\mathbf{w}_{q}(0)$ of (15) as the initial weight vector suffers from slow convergence and high steady-state MSE misadjustment, because $\mathbf{x}(k)$ is highly correlated. In the work [20], a GNCMA+SDD algorithm was proposed to adjust the STE (12) with $\mathbf{w}_{q}(0)$ of $(15)$ as the initial weight vector, which is capable of converging fast and accurately to the optimal MMSE STE solution under a stationary environment.

In the GN-CMA+SDD based STE, the STE's weight vector is split into two parts, yielding $\mathbf{w}_{q}(k)=\mathbf{w}_{q, c}(k)+$ $\mathbf{w}_{q, d}(k)$. The initial $\mathbf{w}_{q, c}$ and $\mathbf{w}_{q, d}$ are simply set to $\mathbf{w}_{q, c}(0)=\mathbf{w}_{q, d}(0)=0.5 \mathbf{w}_{q}(0)$, where $\mathbf{w}_{q}(0)$ is given by (15). A GN algorithm uses the inverse of the autocorrelation matrix of $\mathbf{x}(k)$ to modify the stochastic gradient [22], [23]. Just like in the RLS algorithm, this inverse matrix can be updated recursively according to [10]

$$
\mathbf{P}(k)=\lambda^{-1} \mathbf{P}(k-1)-\lambda^{-1} \mathbf{g}(k) \mathbf{x}^{\mathrm{H}}(k) \mathbf{P}(k-1)
$$

with

$$
\mathbf{g}(k)=\frac{\lambda^{-1} \mathbf{P}(k-1) \mathbf{x}(k)}{1+\lambda^{-1} \mathbf{x}^{\mathrm{H}}(k) \mathbf{P}(k-1) \mathbf{x}(k)},
$$

where $0<\lambda<1$ is the forgetting factor [10]. The initial $\mathbf{P}(0)$ can be set to $\mathbf{P}(0)=\left(\mathbf{X}_{K} \mathbf{X}_{K}^{\mathrm{H}}\right)^{-1}$.

The weight vector $\mathbf{w}_{q, c}$ is updated using the GN-CMA according to

$$
\mathbf{w}_{q, c}(k+1)=\mathbf{w}_{q, c}(k)+\mu_{\mathrm{CMA}} \mathbf{P}(k) \varepsilon^{*}(k) \mathbf{x}(k)
$$




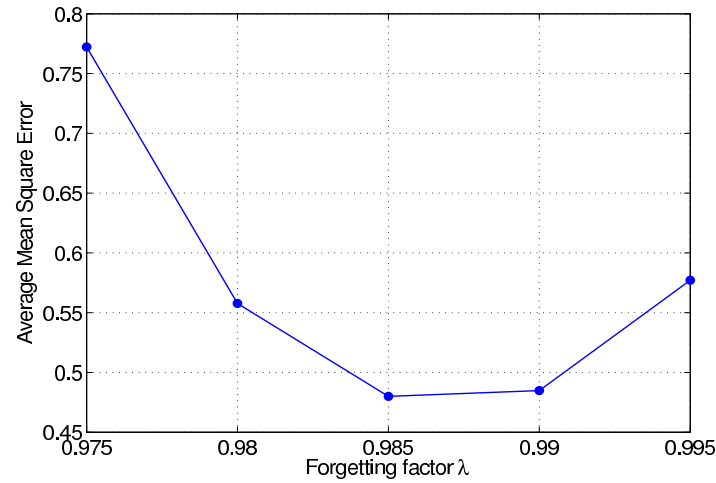

(a)

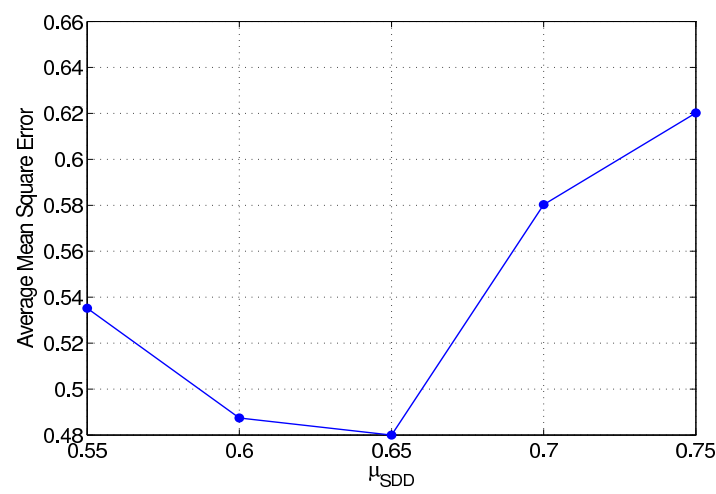

(c)

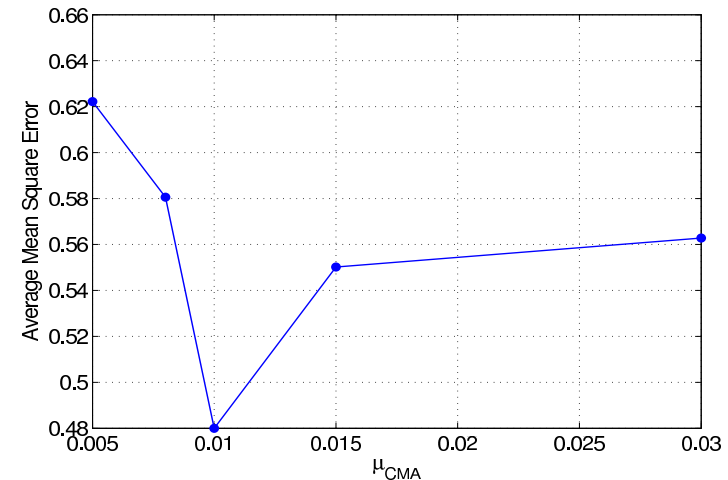

(b)

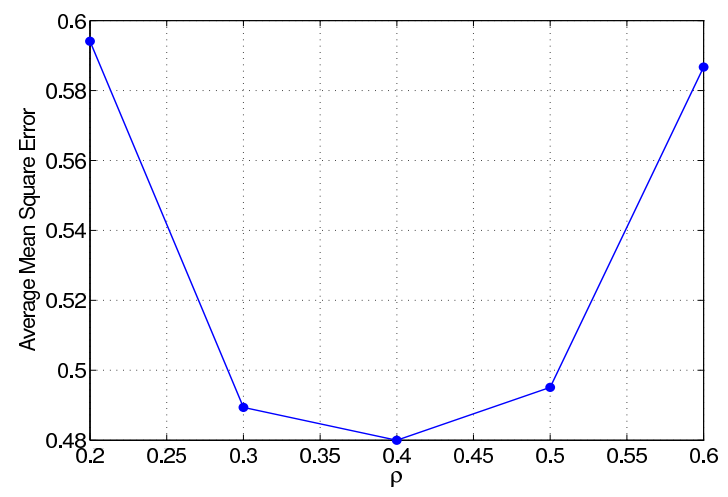

(d)

Fig. 1. Influence of (a) the forgetting factor $\lambda$ with $\mu_{\mathrm{CMA}}=0.01, \mu_{\mathrm{SDD}}=0.65$ and $\rho=0.4$, (b) the CMA step size $\mu_{\mathrm{CMA}}$ with $\lambda=0.985$, $\mu_{\mathrm{SDD}}=0.65$ and $\rho=0.4$, (c) the SDD step size $\mu_{\mathrm{SDD}}$ with $\lambda=0.985, \mu_{\mathrm{CMA}}=0.01$ and $\rho=0.4$, and (d) the cluster width $\rho$ with $\lambda=0.985$, $\mu_{\mathrm{CMA}}=0.01$ and $\mu_{\mathrm{SDD}}=0.65$, to the average MSE of the semi-blind GN-CMA+SDD algorithm, given SNR of $20 \mathrm{~dB}$ and averaged over 50 runs.

with

$$
\varepsilon(k)=y_{q}(k)\left(\Delta-\left|y_{q}(k)\right|^{2}\right),
$$

where $y_{q}(k)=\mathbf{w}_{q}^{\mathrm{H}}(k) \mathbf{x}(k), \Delta=E\left[\left|s_{q}(k)\right|^{4}\right] / E\left[\left|s_{q}(k)\right|^{2}\right]$ and $\mu_{\mathrm{CMA}}$ is the step size of the CMA. This GN-CMA algorithm reduces to the conventional SG-CMA [24], [25] if $\mathbf{P}(k)$ is replaced with an identity matrix. It is wellknown that the step size for the SG-CMA must be chosen sufficiently small to avoid divergence, particularly in a highly correlated signal environment. By contrast, the step size of the GN-CMA algorithm can be set to a value much larger than the step size of the SG-CMA counterpart.

The weight vector $\mathbf{w}_{q, d}$ is updated using the GN-SDD scheme, which is now summarised. The complex phasor plane is divided into the $M / 4$ rectangular regions, and each region $\mathcal{S}_{i, l}$ contains four symbol points as defined by

$$
\mathcal{S}_{i, l}=\left\{s_{r, m}, r=2 i-1,2 i, m=2 l-1,2 l\right\},
$$

where $1 \leq i, l \leq \sqrt{M} / 2$. If the STE's output $y_{q}(k) \in \mathcal{S}_{i, l}$, a local approximation of the marginal probability density function (PDF) of $y_{q}(k)$ is given by [21], [26]

$$
\hat{p}\left(\mathbf{w}_{q}(k), y_{q}(k)\right) \approx \sum_{r=2 i-1}^{2 i} \sum_{m=2 l-1}^{2 l} \frac{1}{8 \pi \rho} e^{-\frac{\left|y_{q}(k)-s_{r, m}\right|^{2}}{2 \rho}},
$$

where $\rho$ is the cluster width associated with the four clusters of each $\mathcal{S}_{i, l}$. The SDD scheme [21], [26], [27] is designed to maximise the local marginal PDF criterion
$J_{\text {LMAP }}\left(\mathbf{w}_{q}(k), k\right)=\rho \log \left(\hat{p}\left(\mathbf{w}_{q}(k), y_{q}(k)\right)\right)$. In particular, the GN-SDD algorithm updates $\mathbf{w}_{q, d}$ according to

$$
\mathbf{w}_{q, d}(k+1)=\mathbf{w}_{q, d}(k)+\mu_{\mathrm{SDD}} \mathbf{P}(k) \frac{\partial J_{\mathrm{LMAP}}\left(\mathbf{w}_{q}(k), k\right)}{\partial \mathbf{w}_{q, d}},
$$

where $\mu_{\mathrm{SDD}}$ is the step size of the SDD, and

$$
\begin{aligned}
\frac{\partial J_{\mathrm{LMAP}}\left(\mathbf{w}_{q}(k), k\right)}{\partial \mathbf{w}_{q, d}}= & \frac{1}{Z_{N}} \sum_{r=2 i-1}^{2 i} \sum_{m=2 l-1}^{2 l} e^{-\frac{\left|y_{q}(k)-s_{r, m}\right|^{2}}{2 \rho}} \\
& \times\left(s_{r, m}-y_{q}(k)\right)^{*} \mathbf{x}(k)
\end{aligned}
$$

with

$$
Z_{N}=\sum_{r=2 i-1}^{2 i} \sum_{m=2 l-1}^{2 l} e^{-\frac{\left|y_{q}(k)-s_{r, m}\right|^{2}}{2 \rho}}
$$

This GN-SDD algorithm reduces to the SG-SDD algorithm of [21], [26] by replacing $\mathbf{P}(k)$ with an identity matrix. Note that, for the SG-SDD algorithm, the step size $\mu_{\mathrm{SDD}}$ has significant influence on the performance of the algorithm, as too large value of $\mu_{\mathrm{SDD}}$ results in divergence while too small value of $\mu_{\mathrm{SDD}}$ leads to slow convergence. By contrast, for the GN-SDD algorithm, $\mu_{\mathrm{SDD}}$ can be set to a much larger value than for the step size of the SG-SDD counterpart. The performance of the GN-SDD algorithm is not overly sensitive to the cluster width $\rho$, defined in the context of the local PDF (21), as in the case of the SG-SDD [19], [21], [26]. 


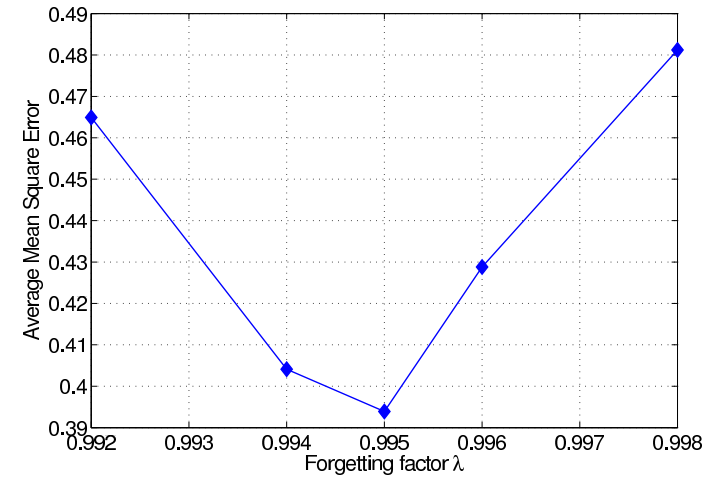

Fig. 2. Influence of the forgetting factor $\lambda$ to the average MSE of the training-based RLS algorithm, given SNR of $20 \mathrm{~dB}$ and averaged over 50 runs.

\section{Simulation Study}

The mean square error (MSE) value for the STE of (12) with the weight vector $\mathbf{w}_{q}(k)$ can be expressed by

$$
\begin{gathered}
J_{\mathrm{MSE}}\left(\mathbf{w}_{q}(k), k\right)=\sigma_{s}^{2}\left(1-\mathbf{w}_{q}^{\mathrm{H}}(k) \mathbf{C}_{\mid q_{\eta}}(k)-\mathbf{w}_{q}^{\mathrm{T}}(k) \mathbf{C}_{\mid q_{\eta}}^{*}(k)\right) \\
+\sigma_{s}^{2} \mathbf{w}_{q}^{\mathrm{H}}(k)\left(\mathbf{C}(k) \mathbf{C}^{\mathrm{H}}(k)+\frac{2 \sigma_{n}^{2}}{\sigma_{s}^{2}} \mathbf{I}\right) \mathbf{w}_{q}(k)
\end{gathered}
$$

where I denotes the $N_{\mathrm{STE}} \times\left(Q \cdot\left(D+n_{C}-1\right)\right)$ dimensional identity matrix, $q_{\eta}=(q-1)\left(D+n_{C}-1\right)+\left(\tau_{q}+1\right)$ and $\mathbf{C}_{\mid i}$ the $i$ th column of $\mathbf{C}$. Then the average MSE

$$
J_{\mathrm{AMSE}}(\mathbf{W}(k), k)=\frac{1}{Q} \sum_{q=1}^{Q} J_{\mathrm{MSE}}\left(\mathbf{w}_{q}(k), k\right),
$$

over all the $Q$ users can be used to investigate the tracking performance of an adaptive STE, where $\mathbf{W}(k)=$ $\left[\mathbf{w}_{1}(k) \mathbf{w}_{2}(k) \cdots \mathbf{w}_{Q}(k)\right]$ denotes the weight matrix of all the $Q$ STEs. Since $J_{\mathrm{MSE}}\left(\mathbf{w}_{q}(k), k\right)$ is a stochastic quantity, whose value depends on the channel realisation, averaging over a number of different runs is necessary. Ultimately, the SER can be simulated to assess the STE's performance.

The simulated system supported $Q=3$ users with the 16-QAM modulation and $P=4$ receive antennas. Each of the $P \cdot Q=12$ CIRs had $n_{C}=3$ taps. The STE's temporal filter order was $D=5$. The three decision delays of the three STEs were set to $\tau_{1}=\tau_{2}=\tau_{3}=2$. Note that there was a trade off in choosing an appropriate temporal filter length $D$. A larger $D$ offered potentially better performance but resulted in longer adaptation period and higher steady-state misadjustment, which was a particular problem for timevarying channels. As the system was under continuously fluctuating fading, the CIRs were changed at each $k$. The RLS based STE benchmark kept training continuously, which was obviously impractical to implement in reality but offered a lower bound of the system's achievable performance.

In the simulation, the normalised Doppler frequency was $f_{d}=10^{-5}$, and the number of the training symbols was $K=24$, which was slightly larger than the STE's dimension $N_{\mathrm{STE}}$. Appropriate algorithmic parameters were found empirically. According to the results plotted in Fig. 1, the step sizes were set to $\mu_{\mathrm{CMA}}=0.01$ and $\mu_{\mathrm{SDD}}=0.65$, while the

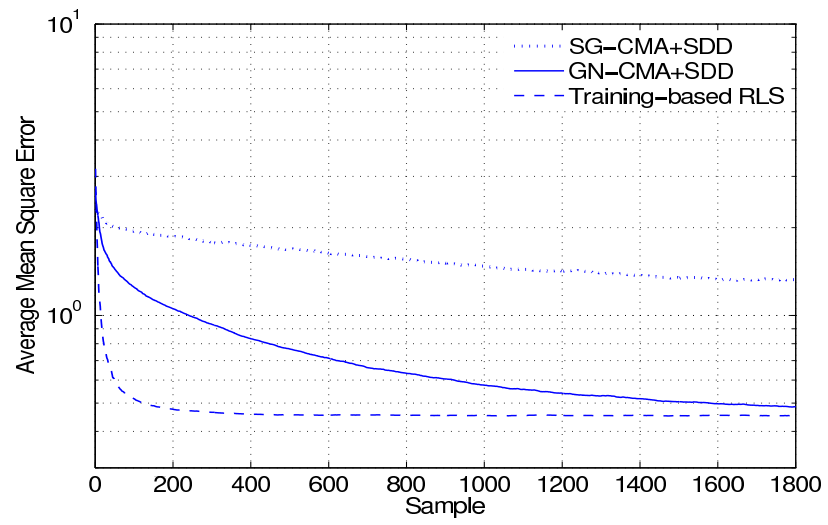

Fig. 3. Tracking performance comparison of the training-based RLS, semiblind SG-CMA+SDD and semi-blind GN-CMA+SDD based STEs, in terms of the average MSE, given $\mathrm{SNR}=20 \mathrm{~dB}$ and averaged over 50 runs.

cluster width and forgetting factor were chosen as $\rho=0.4$ and $\lambda=0.985$, for the GN-CMA+SDD algorithm. We also tested the SG-CMA+SDD based STE, whose algorithmic parameters were chosen to be the step sizes $\mu_{\mathrm{CMA}}=5 \times 10^{-6}$ and $\mu_{\mathrm{SDD}}=0.0003$, while the cluster width $\rho=0.4$, based on a similar experimental procedure. For the training-based RLS algorithm the forgetting factor was set to $\lambda=0.995$ according to the results depicted in Fig. 2.

Fig. 3 plots the learning curves of the training-based RLS, semi-blind SG-CMA+SDD and semi-blind GN-CMA+SDD based STEs, in terms of the average MSE over all the $Q=3$ users, given the SNR value of $20 \mathrm{~dB}$. It can be seen from Fig. 3 that the tracking performance of the semiblind GN-CMA+SDD algorithm was close to that of the continuously training-based RLS algorithm. Fig. 3 also confirms that the semi-blind SG-CMA+SDD algorithm suffered from slow convergence and excessively high steady-state misadjustment in the highly dispersive and fading MIMO signal environment. The SERs of the training-based RLS, semi-blind SG-CMA+SDD and semi-blind GN-CMA+SDD based STEs are depicted in Fig. 4 (a) to (c), for the users one to three, respectively. The results obtained in Fig. 4 demonstrate that the SER performance of the semi-blind GNCMA+SDD based STE was close to that of the continuously training-based RLS STE. This is very significant, considering the fact that the continuously training-based RLS STE is impossible to realise and its SER offers a low bound of the system's achievable performance.

\section{Conclusions}

A semi-blind STE has been investigated for frequency selective Rayleigh fading MIMO systems that employ high throughput QAM signalling. A minimum number of training symbols, approximately equal to the dimension of the STE, is used to provide a rough LS estimate of the STE weight vector for the initialisation. A concurrent GN-CMA+SDD blind adaptive scheme is then adopted to adapt the STE. This semi-blind STE scheme has a complexity similar to that of the training-based RLS algorithm. Our simulation results involving a continuously fluctuating fading MIMO channel have demonstrated that the tracking performance of 


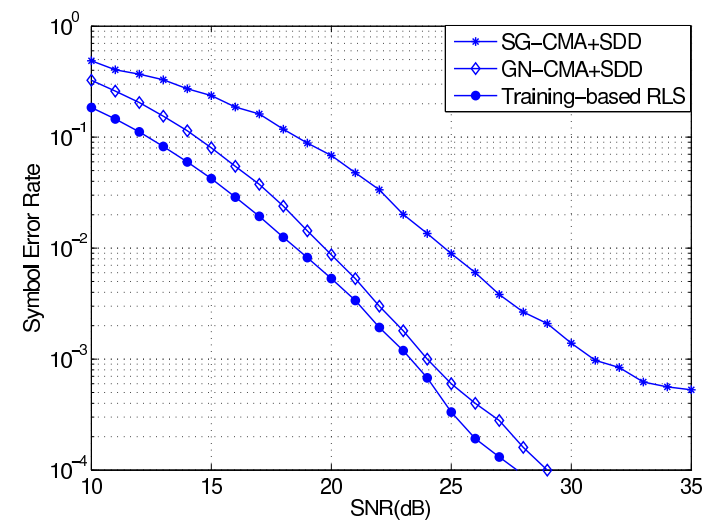

(a)

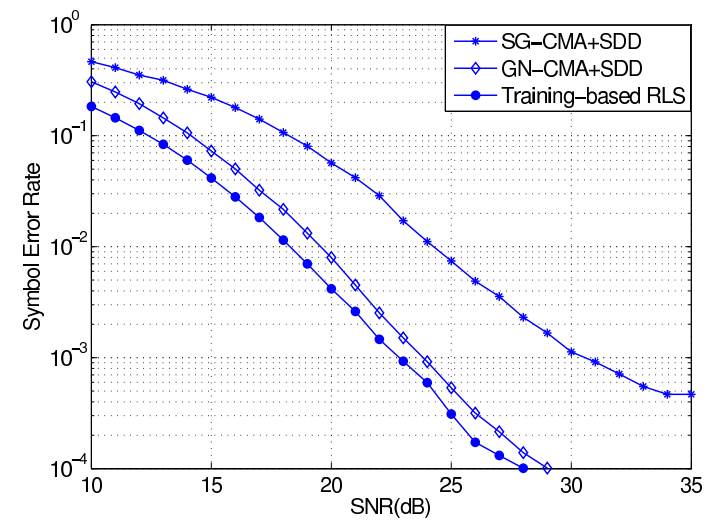

(b)

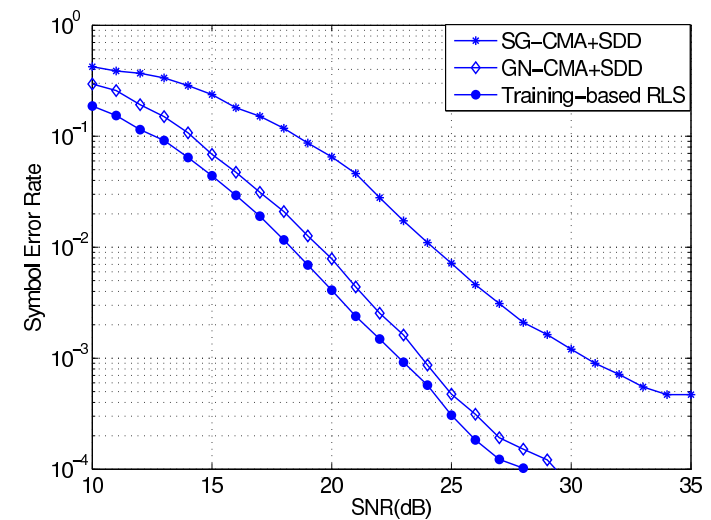

(c)

Fig. 4. SER performance comparison of the training-based RLS, semiblind SG-CMA+SDD and semi-blind GN-CMA+SDD based STEs: (a) user one, (b) user two, and (c) user three.

this semi-blind GN-CMA+SDD algorithm is close to that of the continuously training-based RLS algorithm.

\section{REFERENCES}

[1] U. Trautwein, G. Sommerkorn and R. S. Thomä, "A simulation study on space-time equalization for mobile broadband communication in an industrial indoor environment," in Proc. VTC 1999-Spring, vol. 1, (Houston, TX), pp. 511-515, May 16-20, 1999.

[2] U. Trautwein, D. Hampicke, G. Sommerkorn and R. S. Thomä, "Performance of space-time processing for ISI- and CCI-suppression in industrial scenarios," in Proc. VTC 2000-Spring, vol. 3, (Tokyo, Japan), pp. 1894-1898, May 15-18, 2000.

[3] A. Lozano and C. Papadias, "Layered space-time receivers for frequency-selective wireless channels," IEEE Trans. Communications, vol. 50, pp. 65-73, January 2002.
[4] X. Zhu and R. D. Murch, "Layered space-time equalization for wireless MIMO systems," IEEE Trans. Wireless Communications, vol. 2, pp. 1189-1203, November 2003.

[5] S. Chen, A. Livingstone and L. Hanzo, "Minimum bite-error rate design for space-time equalization-based multiuser detection," IEEE Trans. Communications, vol. 54, pp. 824-832, May 2006.

[6] S. Chen, L. Hanzo and A. Livingstone, "MBER Space-time decision feedback equalization assisted multiuser detection for multiple antenna aided SDMA systems," IEEE Trans. Signal Processing, vol. 54, pp. 3090-3098, August 2006.

[7] Y. Lee and W.-R. Wu, "Adaptive decision feedback space-time equalization with generalized sidelobe cancellation," IEEE Trans. Vehicular Technology, vol. 57, pp. 2894-2906, September 2008.

[8] L. Hanzo, S. X. Ng, T. Keller and W. Webb, Quadrature Amplitude Modulation: From Basics to Adaptive Trellis-Coded, Turbo-Equalised and Space-Time Coded OFDM, CDMA and MC-CDMA Systems. Chichester, UK: John Wiley and IEEE Press, 2004.

[9] IEEE 802.16, Air Interface for Fixed Broadband Wireless Access System, Section 8. PHY, 2004.

[10] S. Haykin, Adaptive Filter Theory. Upper Saddle Rever, NJ: Prentice Hall, 3rd ed., 1996.

11] L. Tang, R. W. Liu, V. C. Soon and Y. F. Huang, "Indeterminacy and identifiability of blind identification," IEEE Trans. Circuits and Systems, vol. 38, pp. 499-509, May 1991.

[12] Y. Inouye and R. W. Liu, "A system-theoretic foundation for blind equalization of an FIR MIMO channel system," IEEE Trans. Circuits and Systems Part I: Fundamental Theory and Applications, vol. 49, pp. 425-436, April 2002.

[13] A. Medles and D. T. M. Slock, "Semiblind channel estimation for MIMO spatial multiplexing systems," in Proc. VTC2001-Fall, vol. 2, (Atlantic City, NJ), pp. 1240-1244, October 7-11, 2001

[14] C. Cozzo and B. L. Hughes, "Joint channel estimation and data detection in space-time communications," IEEE Trans. Communications, vol. 51, pp. 1266-1270, August 2003.

[15] T. Wo, P. A. Hoeher, A. Scherb and K. D. Kammeyer, "Performance analysis of maximum-likelihood semiblind estimation of MIMO channels," in Proc. VTC2006-Spring, vol. 4, (Melbourne, Australia), pp. 1738-1742, May 7-10, 2006.

[16] A. K. Jagannatham and B. D. Rao, "Whitening-rotation-based semiblind MIMO channel estimation," IEEE Trans. Signal Processing, vol. 54, pp. 861-869, March 2006.

17] Z. Ding, T. Ratnarajah and C. F. N. Cowan, "HOS-based semi-blind spatial equalization for MIMO Rayleigh fading channels," IEEE Trans. Signal Processing, vol. 56, pp. 248-255, January 2008.

[18] M. Abuthinien, S. Chen and L. Hanzo, "Semi-blind joint maximum likelihood channel estimation and data detection for MIMO systems," IEEE Signal Processing Letters, vol. 15, pp. 202-205, 2008.

[19] S. Chen, W. Yao and L. Hanzo, "Semi-blind adaptive spatial equalisation for MIMO systems with high-order QAM signalling," IEEE Trans. Wireless Communications, vol. 7, pp. 4486-4491, November 2008.

[20] S. Chen, L. Hanzo and H.-T. Cheng, "Semi-blind gradient-Newton CMA and SDD algorithm for MIMO space-time equalisation," in Proc. Globecom 2009, (Honolulu, Hawaii), p. 5 pages, No.30-Dec.4, 2009.

[21] S. Chen and E. S. Chng, "Concurrent constant modulus algorithm and soft decision directed scheme for fractionally-spaced blind equalization," in Proc. ICC 2004, vol. 4, (Paris, France), pp. 2342-2346, June 20-24, 2004.

[22] P. S. R. Diniz, Adaptive Filtering: Algorithms and Practical Implementation. Boston, MA: Kluwer Academic Publishers, 2nd ed., 2003.

23] R. C. de Lamare and R. Sampaio-Neto, "Adaptive MBER decision feedback multiuser receivers in frequency selective fading channels," IEEE Communications Letters, vol. 7, pp. 73-75, February 2003.

24] D. Godard, "Self-recovering equalization and carrier tracking in twodimensional data communication systems," IEEE Trans. Communications, vol. COM-28, pp. 1867-1875, November 1980.

[25] J. R. Treichler and B. G. Agee, "A new approach to multipath correction of constant modulus signals," IEEE Trans. Acoustics, Speech and Signal Processing, vol. ASSP-31, pp. 459-472, April 1983.

[26] S. Chen, T. B. Cook and L. C. Anderson, "A comparative study of two blind FIR equalizers,", Digital Signal Processing, vol. 14, pp. 18-36, January 2004.

[27] S. Chen and L. Hanzo, "Fast converging semi-blind space-time equalisation for dispersive QAM MIMO systems," IEEE Trans. Wireless Communications, vol. 8, pp. 3969-3974, August 2009. 